\title{
Acquisition of oral microbiota is driven by environment, not host genetics
}

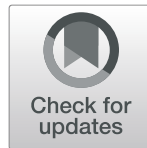

Chiranjit Mukherjee ${ }^{1}$, Christina O. Moyer ${ }^{1}$, Heidi M. Steinkamp ${ }^{1}$, Shahr B. Hashmi ${ }^{1}$, Clifford J. Beall', Xiaohan Guo ${ }^{2}$, $\mathrm{Ai} \mathrm{Ni}^{2}$, Eugene J. Leys ${ }^{1}$ and Ann L. Griffen ${ }^{1 *}$

\begin{abstract}
Background: The oral microbiota is acquired very early, but the factors shaping its acquisition are not well understood. Previous studies comparing monozygotic (MZ) and dizygotic (DZ) twins have suggested that host genetics plays a role. However, all twins share an equal portion of their parent's genome, so this model is not informative for studying parent-to-child transmission. We used a novel study design that allowed us to directly examine the genetics of transmission by comparing the oral microbiota of biological versus adoptive mother-child dyads.
\end{abstract}

Results: No difference was observed in how closely oral bacterial community profiles matched for adoptive versus biological mother-child pairs, indicating little if any effect of host genetics on the fidelity of transmission. Both adopted and biologic children more closely resembled their own mother as compared to unrelated women, supporting the role of contact and environment. Mother-child strain similarity increased with the age of the child, ruling out early effects of host genetic influence that are lost over time. No effect on the fidelity of mother-child strain sharing from vaginal birth or breast feeding was seen. Analysis of extended families showed that fathers and mothers were equally similar to their children, and that cohabitating couples showed even greater strain similarity than mother-child pairs. These findings support the role of contact and shared environment, and age, but not genetics, as determinants of microbial transmission, and were consistent at both species and strain level resolutions, and across multiple oral habitats. In addition, analysis of individual species all showed similar results.

Conclusions: The host is clearly active in shaping the composition of the oral microbiome, since only a few of the many bacterial species in the larger environment are capable of colonizing the human oral cavity. Our findings suggest that these host mechanisms are universally shared among humans, since no effect of genetic relatedness on fidelity of microbial transmission could be detected. Instead our findings point towards contact and shared environment being the driving factors of microbial transmission, with a unique combination of these factors ultimately shaping the highly personalized human oral microbiome.

Keywords: Oral microbiome, Acquisition, Strain level, Genetics, Transmission, Human

\footnotetext{
* Correspondence: griffen.1@osu.edu

${ }^{1}$ College of Dentistry, The Ohio State University, Columbus, OH, USA

Full list of author information is available at the end of the article
}

(C) The Author(s). 2021 Open Access This article is licensed under a Creative Commons Attribution 4.0 International License, which permits use, sharing, adaptation, distribution and reproduction in any medium or format, as long as you give appropriate credit to the original author(s) and the source, provide a link to the Creative Commons licence, and indicate if changes were made. The images or other third party material in this article are included in the article's Creative Commons licence, unless indicated otherwise in a credit line to the material. If material is not included in the article's Creative Commons licence and your intended use is not permitted by statutory regulation or exceeds the permitted use, you will need to obtain permission directly from the copyright holder. To view a copy of this licence, visit http://creativecommons.org/licenses/by/4.0/ The Creative Commons Public Domain Dedication waiver (http://creativecommons.org/publicdomain/zero/1.0/) applies to the data made available in this article, unless otherwise stated in a credit line to the data. 


\section{Introduction}

The last decade has seen rapid growth in understanding the role of the human microbiome in health and disease. Foundational studies across multiple body sites have shown that the adult human microbiota consists of a shared, limited set of niche-specific species [1,2]. Our previous work exploring the assembly of oral microbiota from birth through the first year of life showed that the early childhood microbiota is acquired in an ordered sequence, with common oral species shared between children and mothers [3]. At the strain level, however, each individual harbors a highly personalized microbiota $[4$, 5]. The role of intrinsic and extrinsic factors in acquisition of this individualized microbiome is yet not well understood. Given that strains have been found to be shared among family members [6], a possible intrinsic factor influencing microbiota acquisition could be host genetics shared between parents and offspring.

A number of previous investigations into the interactions of microbes and host genetics have compared microbial communities of monozygotic (MZ) twins and dizygotic (DZ) twins. Two of these studies have shown the oral microbiota of MZ twins to be slightly more similar than that of DZ twins, suggesting that host genetics influences microbial community composition [7, 8]. These studies also identified taxa that are more likely to be "heritable." Microbial "heritability" could have far-reaching consequences for health, since the inheritance of a dysbiotic community could confer increased risk for diseases with a microbial etiology. Yet, no studies have specifically examined the fidelity with which the human oral microbiota are passed from parents to offspring.

A major deterrent to studying the role of shared genetics in parent to child microbial transmission has been the lack of a suitable case-control model. Both MZ and DZ twins share an equal portion of their parent's genome and are therefore not informative for studying direct parent to offspring transmission. To specifically examine the contribution of genetics to the fidelity with which microbial species and strains are passed from parents to offspring, we used a novel study design comparing genetically related and unrelated mother-child pairs. The fraction of shared species and strains between children and their biological mothers, who share half of their genomes, was compared with that of children and their adoptive mothers, who had no genetic relationship. Extensive metadata was collected to examine potential confounders such as breastfeeding and birth mode. Given that humans share a core oral microbiota at the species level [9], subspecies or strain level techniques are required to accurately track microbial transmission between individuals. We have developed a highthroughput strategy for sub-species/strain characterization of bacterial communities by targeting the ribosomal intergenic spacer region (ISR), and this approach has revealed highly personalized profiles among adults [5]. We employed this approach for sub-species analysis, along with 16S rRNA gene sequencing for species analysis. Microbial community composition is known to vary among distinct niches within the oral cavity that are important in oral disease. Therefore, to comprehensively profile the oral microbiota, we sampled three distinct oral habitats-soft tissue and saliva, and both supragingival and subgingival plaque biofilm.

Using this multi-habitat, multi-resolution approach, we comprehensively profiled oral microbial communities in parents and children. We detected no effect of genetic relationship on fidelity of transmission at either the species or strain level, and no effect in any of the three biologically important oral niches. Our findings suggest that contact and shared environment, not genetics, determine the transmission of oral microbes. An extended dataset showing high similarity between spouses further supported this observation.

\section{Methods}

Inclusion/exclusion criteria, exam and sampling

Adoptive and biological mother-child dyads were enrolled to allow determination of the effect of genetic relatedness on the fidelity of oral bacterial transmission. IRB approval was obtained for this study, parents provided written consent and children over 7 years of age provided assent. Adoptive mother-child dyads were recruited through adoption agencies. The biological group was recruited to match the adoptive group on child's age, and parent's socioeconomic status. Only children adopted immediately at birth and unrelated to the adoptive family were included to minimize transmission of bacteria from the biological mother. Only genetic birth mothers were included in the biological group, and fathers and siblings from this group were also sampled when available. For both biological and adoptive groups, the minimum age for children was 3 months to allow the establishment of an oral bacterial community, while twelve years of age was the maximum. Exclusion criteria for all subjects were chronic disease affecting the oral cavity or immune system, and/or early onset periodontitis.

\section{Examination and history}

A dental exam was conducted and recorded for both children and mothers that included caries, gingivitis, periodontitis, and plaque levels. A medical and social history was also obtained, including breastfeeding history and delivery mode.

\section{Sample collection and processing}

Sampling was conducted at least $1 \mathrm{~h}$ after home oral hygiene or consuming food or drink. Saliva and soft tissue were sampled with a Copan swab placed in the right 
lingual vestibule for $30 \mathrm{~s}$ and then swabbed into both buccal vestibules and across the tongue. Sterile microbrushes were used to collect supragingival plaque from the buccal surfaces of all teeth in the mandibular right quadrant, and sterile paper points were then inserted into the mesial sulcus of each of these teeth to obtain subgingival samples. Samples were placed separately in labeled tubes containing $200 \mu \mathrm{L}$ ATL buffer (Qiagen, USA) and stored under refrigeration until transported to the lab for storage at $-20{ }^{\circ} \mathrm{C}$. Bacterial genomic DNA was extracted using QIAamp DNA Mini Kit (Qiagen, USA), using an optimized protocol as described in Mukherjee et all 2018 [5].

\section{Sequencing library preparation}

Two sets of sequencing libraries were prepared, targeting the 16S V1-V3 and 16-23S ISR, as described in Mukherjee et al 2018. Briefly, both the protocols are based on an optimized Illumina 16S Metagenomic Sequencing Library Preparation protocol (Illumina, USA), a two-step process where the target region is PCR amplified with gene-specific primers and then indexing barcodes are added through a second round of amplification. An optimization was made to the molecular protocol for the 16-23S ISR library such that only the ISR fragment was amplified as target, excluding the adjacent $16 \mathrm{~S}$ region. This was achieved using the rD1f primer (5'-GGCTGG ATCACCTCCTT) [10] in place of the 1237F primer in the original protocol. The 16S V1-V3 libraries were sequenced on the Illumina MiSeq Platform with 300 base pair paired-end chemistry. The ISR libraries were sequenced on the Illumina HiSeq 2500 platform, with 250 base pair paired-end chemistry. Illumina MiSeq sequencing was used for the $16 \mathrm{~S}$ reads to allow the entire V1V3 region to be sequenced. Given that the average ISR is much smaller length, the shorter read HiSeq platform was chosen for sequencing the ISR reads to take advantage of its greater throughput, allowing a single batch run for all the samples. The mean per sample read count was comparable among the two sequencing methods, at 24427 and 26606 for the ISR and 16S methods, respectively.

\section{Sequence data processing and taxonomic assignment}

Demultiplexed reads from the 16S V1-V3 library were processed as described in Mukherjee et al. 2018 [5]. Briefly, paired reads from the $16 \mathrm{~S}$ library were merged using Mothur [11], quality filtered using Mothur and custom Python scripts, and then mapped against our oral bacteria16S reference database CORE [12] to generate species-level OTU abundance tables. All scripts used in this pipeline are available publicly at https://github. com/cliffbeall/leyslab-amplicon-pipeline.
For the ISR library, denoising and sample inference was performed using DADA2 [13] version 1.10.0 for the unpaired read $1 \mathrm{~s}$ (forward read), as described in Mukherjee et al. 2018 [5], with quality filtering parameters optimized to suit this library. Specifically, parameters used in the DADA2 processing protocol were adjusted for the primers used in this study and overall library quality (trimLeft $=17, \operatorname{maxN}=0$, truncQ $=2$ ). Chimeric reads were removed with DADA2 using the function removeBimeraDenovo (method = "consensus") and low throughput (<5000 input sequences) samples were excluded from the analysis. Samples from multiple oral niches were included for each subject, and ISR Amplicon Sequence Variants (ASVs) present in only one sample were removed as suspected PCR artifacts.

For comparisons among species, ISR ASVs from the soft tissue swab/saliva samples were mapped to the updated Human Oral ISR database [5]. This database now constitutes of over 3000 unique ISR sequences representing close to 300 different species of the most abundant oral bacteria and is publicly available for download (https://github.com/cm0109/ISR_database).

\section{Statistical analysis and visualizations}

Statistical data analysis and visualizations were performed in RStudio version 1.1.456, with $\mathrm{R}$ version 3.5.1. ASV and species-OTU tables were rarefied using the $\mathrm{R}$ function rrarefy from the vegan [14] package, using row minimums as subsample size. Relevant distance matrices (Bray-Curtis and Jaccard) were generated using the function vegdist, also from package vegan. Non-metric multidimensional scaling (NMDS) ordination was computed using the function metaMDS from the vegan package. Ellipses were drawn at 95\% confidence level for the NMDS plots using stat ellipse function in ggplot2. Distribution of distances between two groups were compared using Wilcoxon rank sum test, with the function stat_compare_means from the package ggpubr [15].

For statistical comparisons between groups where one of the groups consisted of paired subjects and the other of unrelated pairs, a permutation-based method was adopted such that correct distribution of test statistic could be obtained while accounting for dependency among pairs [16]. For this method, the observed test statistic was calculated as the median of the dissimilarity indices of a random subset of all possible unrelated pairs of subjects in the dataset minus the median dissimilarity of paired subjects. The size of the random subset was the same as the number of paired subjects so that the permutation test had comparable power as the Wilcoxon test used in other comparisons. The original pairing of subjects was then randomly permuted, and for each permutation, the new test statistic was calculated in the same way as for the original data. For each comparison, 
1000 such permutations were computed, to obtain the empirical distribution of the test statistic. The two-sided $p$ value was then calculated as the proportion of permutations where the absolute value of the test statistic is larger than or equal to the absolute value of the observed test statistic.

Multiple linear regression analysis was conducted using the function $\mathrm{lm}$ from package stats. Clinically relevant categorical variables recorded as none/mild/moderate/severe were converted to numerical representation for regression analysis using the scale $1 / 2 / 3 / 4$, respectively. For measuring correlation, Spearman's correlation test was applied using function cor.test from package stats, which provided the $p$ value and Spearman's rank correlation coefficient (rho) measure. Fisher's exact test for metadata comparisons were performed using the $\mathrm{R}$ functions fisher.test from package stats.

$\mathrm{R}$ function stars.pval (package gtools v3.5.0) was used to convert numerical $p$ values to star notations. The convention used is as follows: if a $p$ value was less than 0.05 , it was flagged with one star $\left(^{*}\right)$. If a $p$ value was less than 0.01 , it was flagged with two stars (**). If a $p$ value was less than 0.001 , it was flagged with three stars $(* * * *)$.

All visualizations were built using ggplot2 version 3.1.0 [17]. Violin plots and box and whisker plots were generated using ggplot2 functions geom_violin and geom boxplot, respectively. Venn diagrams were plotted using the draw.pairwise.venn function from the $\mathrm{R}$ package VennDiagram (ref). NMDS plots were constructed using geom_points, and vector fitting when applicable were drawn using geom_segment, both from ggplot2. Scatter plots were constructed using the ggplot2 function geom_points, smoothed using the LOESS fit smoothing by function geom_smooth (ggplot2). Bar plots were constructed using the ggplot2 function geom_bar (stat = "identity"). The relevant data and scripts for all analysis performed is available at https://github.com/cm0109/ Adoption_study.

\section{Results}

\section{Multi-niche, multi-resolution study framework}

A multi-niche, multi-resolution approach (Fig. 1) was implemented to compare oral microbial communities of children and adults, to determine mother to child bacterial transmission within genetically related and unrelated families. Subjects recruited for this study included 50 adoptive mother-child- pairs and 55 biological mother-child pairs. The adoptive group included only children adopted at birth by a non-genetic relative. The biological group was matched on age $(p=0.29)$ and socioeconomic status to the adoptive group. Additionally, an extended family dataset of samples was obtained from 23 fathers and 16 siblings of the children in the biological group, allowing comparison of microbial profile similarity among siblings, couples, and child-father pairs. Detailed meta-data including feeding and delivery mode, health measures, and demographics were also collected.

To comprehensively profile microbial communities from multiple niches within the oral cavity, one soft tissue/saliva swab sample, one supragingival plaque sample, and one subgingival plaque sample were collected from each subject, with the exception of predentate children, from whom no tooth-associated sample could be collected. Microbial communities from each sample were independently profiled at both the species and strain level. Species level resolution was achieved through sequencing of the $16 \mathrm{~S} \mathrm{~V} 1-\mathrm{V} 3$ region and mapping to the OSU CORE [12] reference database of oral bacteria, and strain level resolution was achieved by targeted sequencing of the 16-23S Intergenic Spacer Region (ISR) combined with high-resolution processing using DADA2, as shown previously [5].

For strain-level community analysis, the ISR-amplicon library was sequenced using the Illumina HiSeq 2500 platform and processed with DADA2 [13] to generate unique ISR amplicon sequence variants (ISR-ASVs) or ISR-type strains. Seven samples which did not generate sufficient sequences (cutoff 5000 reads) were excluded from analysis. A conservative approach was implemented in calling true biological strain variants by including in the analysis only those ASVs found in more than 1 sample. The final dataset consisted of 778 samples, representing 3865 ASVs. This included 49 adoptive and 54 biological mother-child pairs for the saliva dataset, 46 adoptive and 53 biological pairs for the supragingival dataset, and 44 adoptive and 51 biological pairs for the subgingival dataset. A detailed breakdown of samples in each group is included in Supplementary Table ST1.

For the species-level community analysis, the same samples were used to generate a 16S V1-V3 amplicon library, sequenced on Illumina MiSeq platform, processed using Mothur [11], and species level taxonomy was assigned to the reads using CORE [12] oral $16 \mathrm{~S}$ database. The final quality filtered dataset consisted of 709 samples, representing 581 species-level OTUs. This included 45 adoptive and 48 biological mother-child pairs for the saliva subset, 45 adoptive and 48 biological pairs for the supragingival subset, and 40 adoptive and 46 biological pairs for the subgingival subset. Supplementary Table ST2 lists the details of samples in each group for the 16S V1-V3 dataset.

Non-metric multidimensional scaling (NMDS) ordination using Bray-Curtis (BC) dissimilarities based on community membership was used to compare betadiversity of all the mother and child samples, both at the strain (ISR) and species (16S) levels (Fig. 2). Profiling strain-level communities showed significantly greater separation between the samples compared to species- 


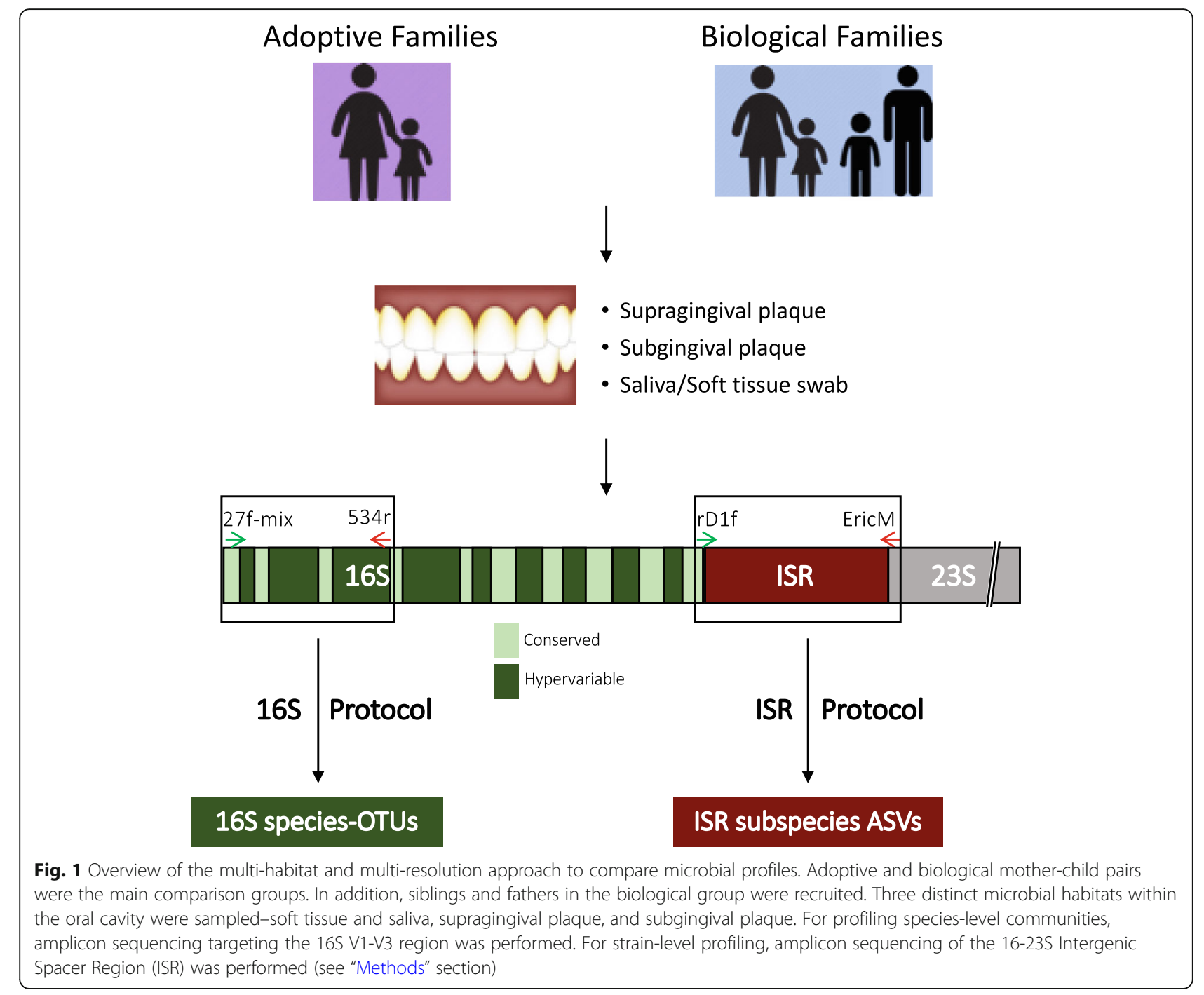

level communities (Supplementary Figure S1). No distinction between the adoptive and biological families in terms of beta diversity was observed (Supplementary Figure S2).

\section{No genetic influence on acquisition of oral bacteria}

Similarity between the microbial profiles of adoptive and biological mother-child pairs was quantified using a distance-based approach. BC dissimilarities were computed for each mother-child pair based on presence/absence of species/strain variants. This index ranges from 0 to 1 , with samples having exactly identical microbial communities scored at 0 and absolutely different communities scored at 1 . We compared the $\mathrm{BC}$ distances between mother-child at both species and strain levels (Fig. 3) using the Wilcoxon rank sum test. Microbial profiles of biological and adopted children were equally similar to their mothers, for all three niches we sampled: saliva/soft tissue, supragingival plaque and subgingival plaque. This was true at both species and strain level resolutions. BC dissimilarities of all possible unrelated mother-child pairings among the samples were also computed. A group containing all possible distances between any unrelated mother-child pairs is often compared with distances between adoptive or biological mother-child pairs using a Wilcoxon test or $t$ test, but this violates a basic assumption of the tests by re-using the same observations multiple times. Therefore, as an alternative to the widely used Wilcoxon test or $t$ test approaches, we used a permutation-based method for statistical comparison between the unrelated group and the adoptive/biological groups (see "Methods" section for details). At the level of strains, all mothers and their own children, regardless of genetic relationship, were significantly more similar to each other than unrelated mother-child pairs. This relationship was not as strong using the lower resolution species-level approach. Similar results were also observed using the Jaccard 


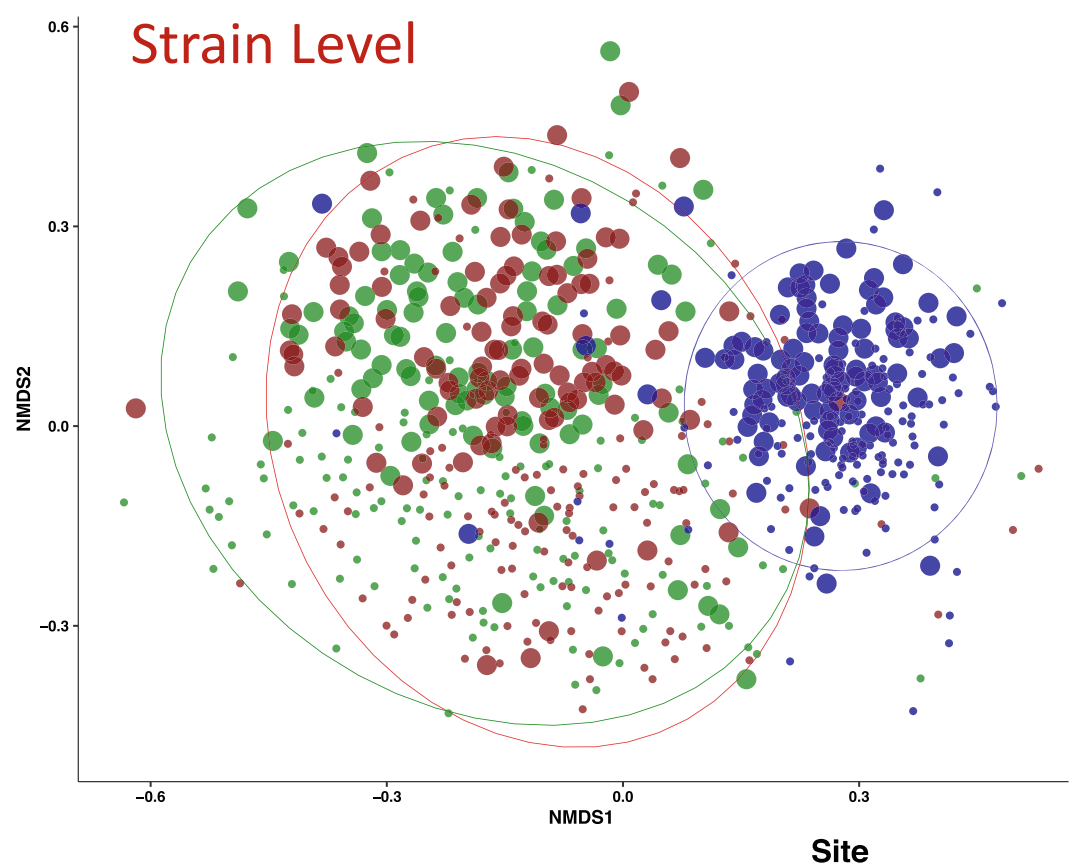

\section{Species Level}

- Soft Tissue/Saliva Swab

- Subgingival Plaque

- Supragingival Plaque

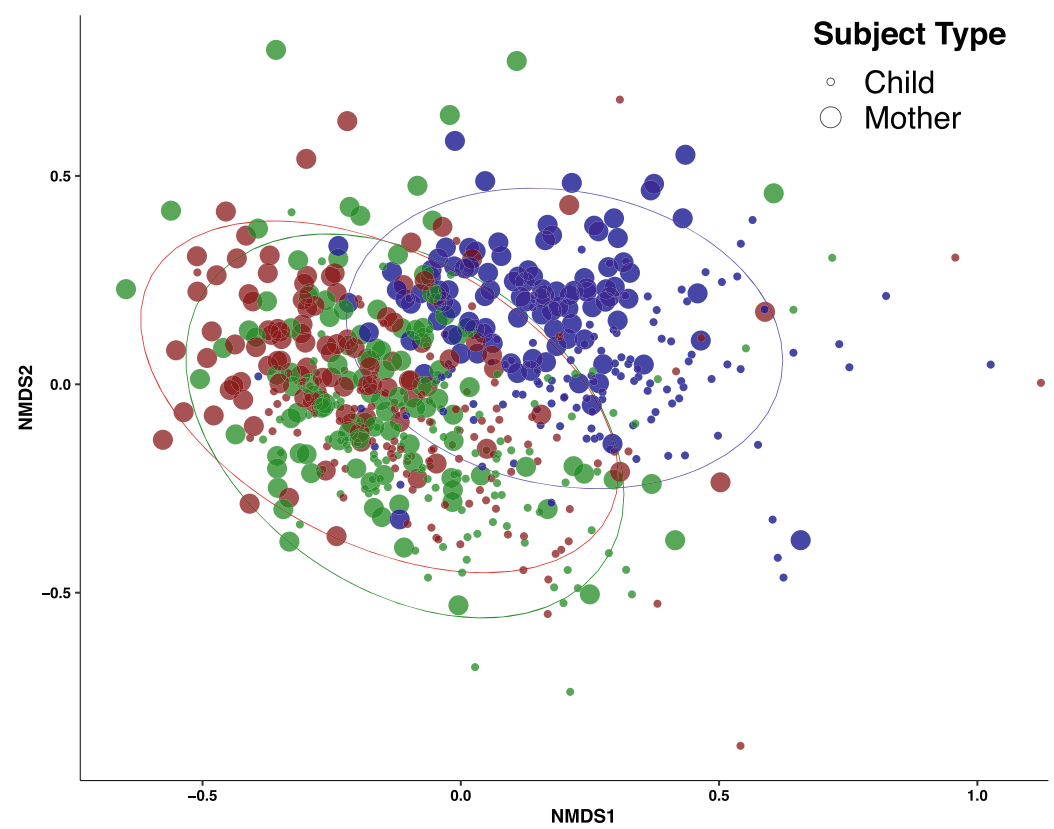

Fig. 2 Beta-diversity comparison among samples by subject type and sampling site, at both species and strain levels. Non-metric multidimensional scaling (NMDS) plots using Bray-Curtis dissimilarities based on community membership, at ISR-strain level (top panel) and 16S Species level (bottom panel) are shown. At the lower resolution (species level) inter-sample distances are smaller as compared to strain level. Ellipses are drawn to show 95\% confidence intervals for each group

dissimilarity index (ISR soft tissue/saliva dataset shown in Supplementary Figure S3). Additionally, when using relative abundance measures in place of presence/absence, similar results for comparisons between adoptive/ biological groups were observed (ISR soft tissue/saliva dataset shown in Supplementary Figure S4). Given that the three sites showed highly similar patterns, and the soft tissue swab/saliva provided the largest dataset for 


\section{Soft Tissue/Saliva}

\section{Supragingival}

\section{Subgingival}
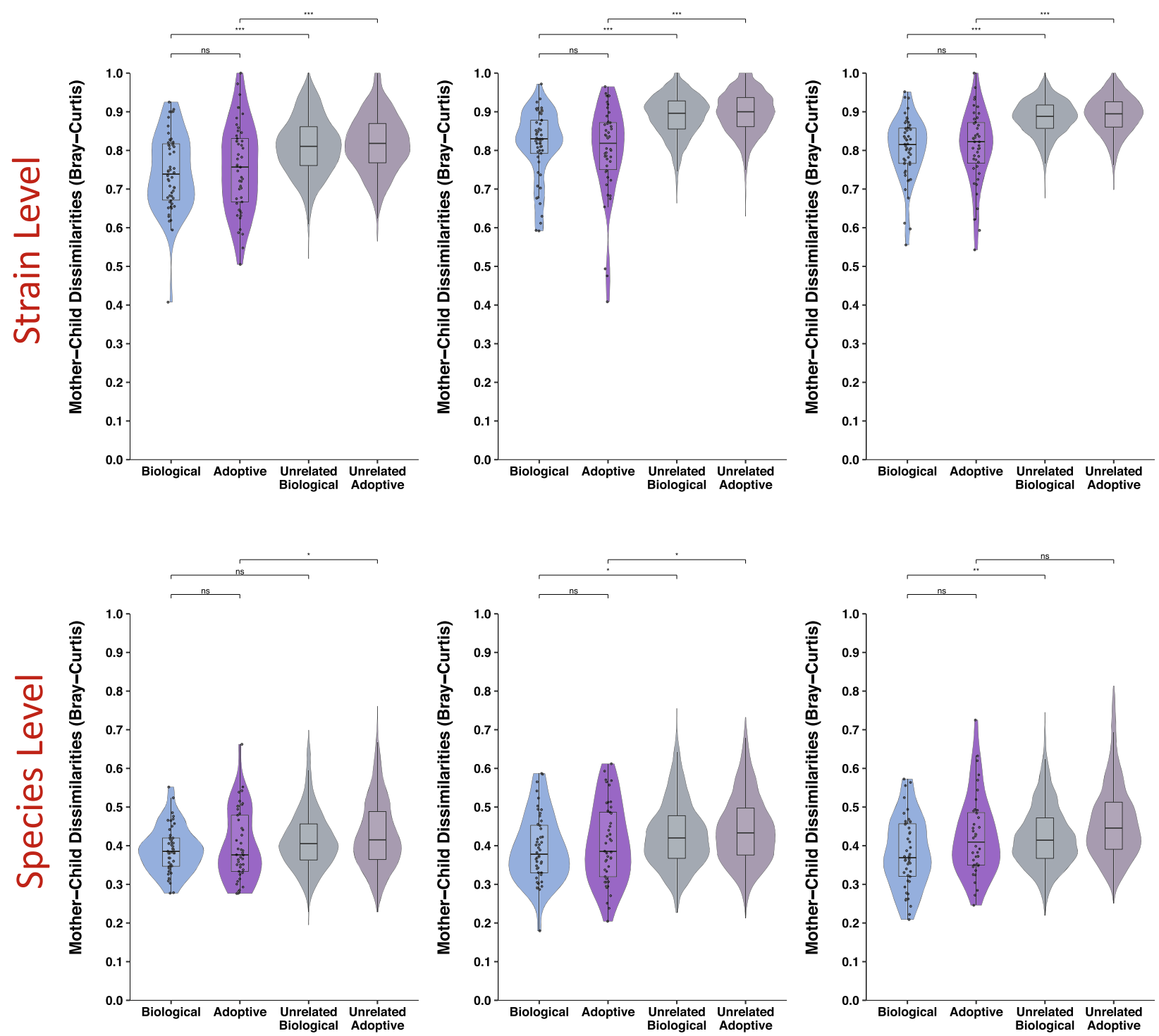

Fig. 3 No influence of genetics on sharing of strains or species between mother and child. Violin plots with embedded box and whisker plots are shown here comparing the distribution of mother-child distances in the biological, adoptive, unrelated biological, and unrelated adoptive groups, for the three sampling sites, at strain (top panel) and species level (bottom panel). No significant difference was observed in the motherchild dissimilarities for the biological and adoptive groups, at either species or strain levels, across the 3 distinct habitats within the oral cavity. Biological vs adoptive statistical comparisons were performed using Wilcoxon rank sum test, and related/unrelated comparisons were performed using a comparable permutation-based test (see "Methods" section). Significance levels: ns: $p>0.05,{ }^{*} p \leq 0.05,{ }^{* *} p \leq 0.01,{ }^{* * *} p \leq 0.001$

our comparisons because it included the predentate children, those samples were used as the primary dataset in subsequent analyses.

We calculated the average number of species and strains shared between mother-child pairs for the soft tissue/saliva samples (Fig. 4). Both adoptive and biological groups shared $44 \%$ of their microbiota at species level, and $15 \%$ at strain level. As expected, the fraction of shared species was much higher than fraction of shared strains, and unrelated mother-child pairs shared four times as many oral species as oral strains. A list of the most widely shared species and their relative abundance in mother-child pairs for both the biological and adoptive families is provided in Supplementary Table ST3. Even though the set of species and strains shared between mothers and children made up $44 \%$ and $15 \%$ of 

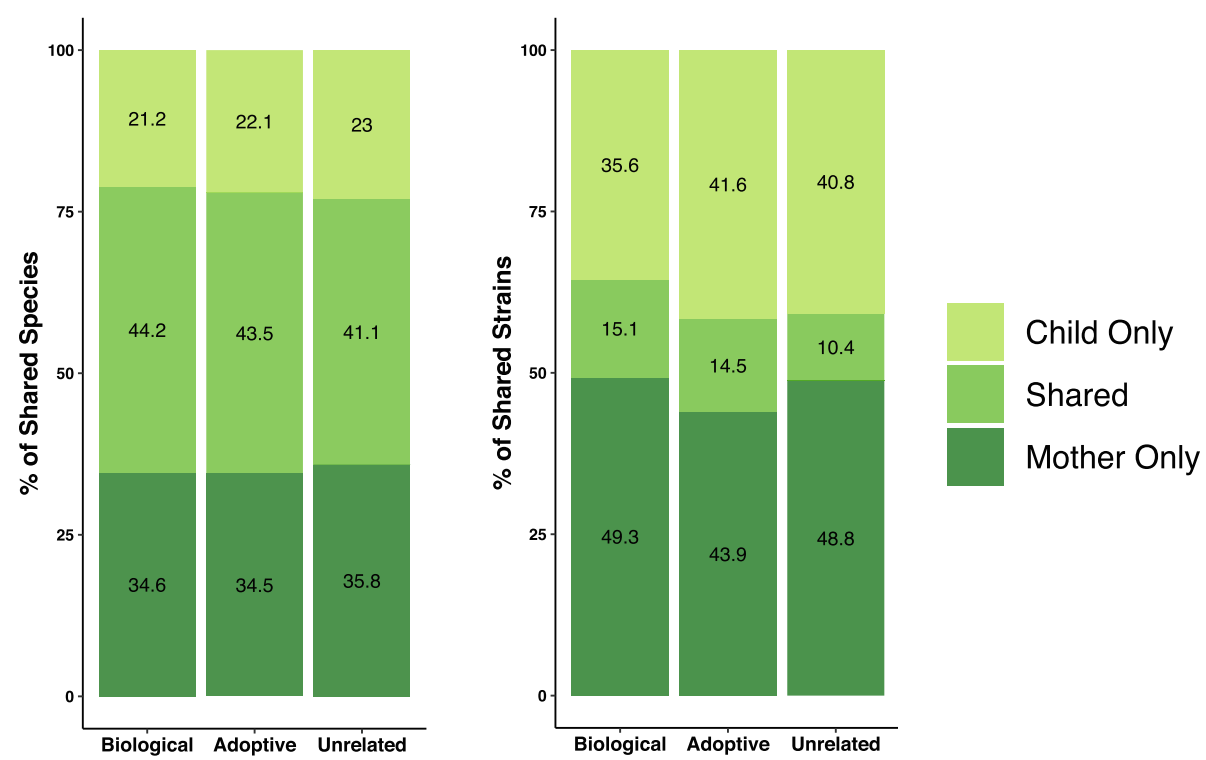

Fig. 4 Adopted and biological mother-child pairs shared similar percentage of species and strains. Stacked bar plots showing mean number of species-OTUs (left) and ISR-strains (right) shared by mother-child pairs in the adoptive, biological and combined unrelated groups for the saliva/ soft tissue swab samples. The percentages of shared species/strains were comparable for the adoptive and biological groups at both resolutions

the total number of species and strain variants, when considering relative abundance, they accounted for $93 \%$ and $48 \%$ of the total communities on average.

\section{Ruling out possible confounders including feeding and delivery mode}

Extensive demographic and clinical data were collected for all subjects and examined to determine if any of these variables significantly influenced mother-child dissimilarities. Targeted recruitment lead to close matching of age, health factors, and socio-economic status between the biological and adopted children (no significant differences). However, the following nine potentially confounding factors for the mother-child dissimilarities were found to be significantly different between the two groups by Wilcoxon rank-sum or Fisher's exact test: child's feeding mode, child's gingivitis level, child's race, child's tongue biofilm level, mother-child race match, mother's age, mother's plaque level, mother's tongue biofilm level, and mother's gingivitis level. To assess the confounding effect, a univariate regression analysis with mother-child distance as the dependent variable and group as the independent variable was performed, and a multiple regression analysis with the unbalanced factors as additional independent variables was also performed. The estimated regression coefficient of the group variable changed substantially from the univariate model (beta $=-0.017, p=0.433$ ) to the multiple regression model (beta $=-0.056, p=0.226$ ). However, the group variable remained non-significant even after adjusting for the confounding effect in the multiple regression model, thereby ruling out any significant confounding effect on the mother-child dissimilarities. Within the biological group neither feeding mode nor delivery mode had a significant impact on mother-child distances for any niche (Supplementary Figure S5).

\section{Extended family comparisons also fail to show influence of genetics}

An extended dataset of the biologically related families that included fathers and siblings was analyzed to further explore the relative contributions of genetics and shared environment. Comparisons of distance between various related and unrelated pairs of subjects for the soft tissue/saliva dataset at strain level is shown in Fig. 5. Cohabitating mother-father couples and sibling pairs showed the greatest oral microbiota resemblance of any pairing examined, sharing significantly more strains than mother-child or father-child pairs. Mothers and fathers were equally similar to their children, and both motherand father-child pairs were significantly more similar than unrelated mother- and father-child pairings. Technical replicates consisting of samples that were processed through the ISR pipeline in duplicates were highly similar. Analysis of the extended family samples from the subgingival and supragingival dataset also produced similar results (Supplementary Figure S6). Taken together, these findings provide further evidence that shared environment and contact, rather than genetic background, is the primary determinant of microbial community structure. 


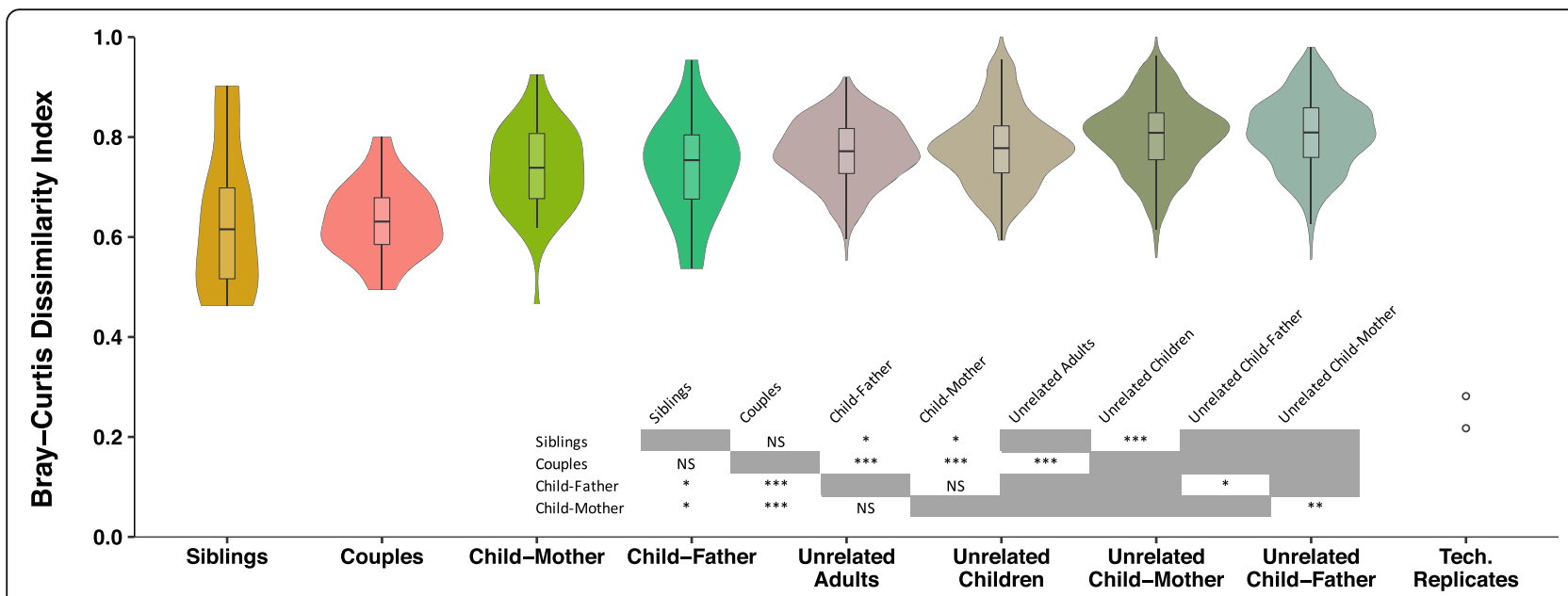

Fig. 5 Shared environment led to greater similarity between individual's oral microbial communities at strain level. Comparing microbial community similarities among different family groups, based on saliva/soft tissue swab samples from the extended biological family dataset. Dissimilarities between individuals were lowest among cohabitating couples and siblings, even compared to child-mother and child-father. Children's oral microbiota were equally similar to their father's, as they were to their mother's. Couples and siblings were more similar to themselves, compared to unrelated adults and unrelated children, respectively. Technical replicates were highly similar to one another. Statistical comparisons were performed using Wilcoxon rank sum test and the previously used permutation test (when including unrelated groups)

Older children's microbiota more similar to their mother's Child's age is known to be a major determinant of oral microbiota composition, but targeted recruitment allowed us to ensure that the child's ages in the adoptive and biologic group were balanced (Fig. 6a). Since our results showed that the biological and adoptive children were equally similar to their mothers, the two groups were combined and examined to determine if child's age had an effect on mother-child dissimilarities. A total of 101 children were considered for this analysis, with an age range of 3 months to 6 years. Contrary to our hypothesis, we saw that younger children's microbial

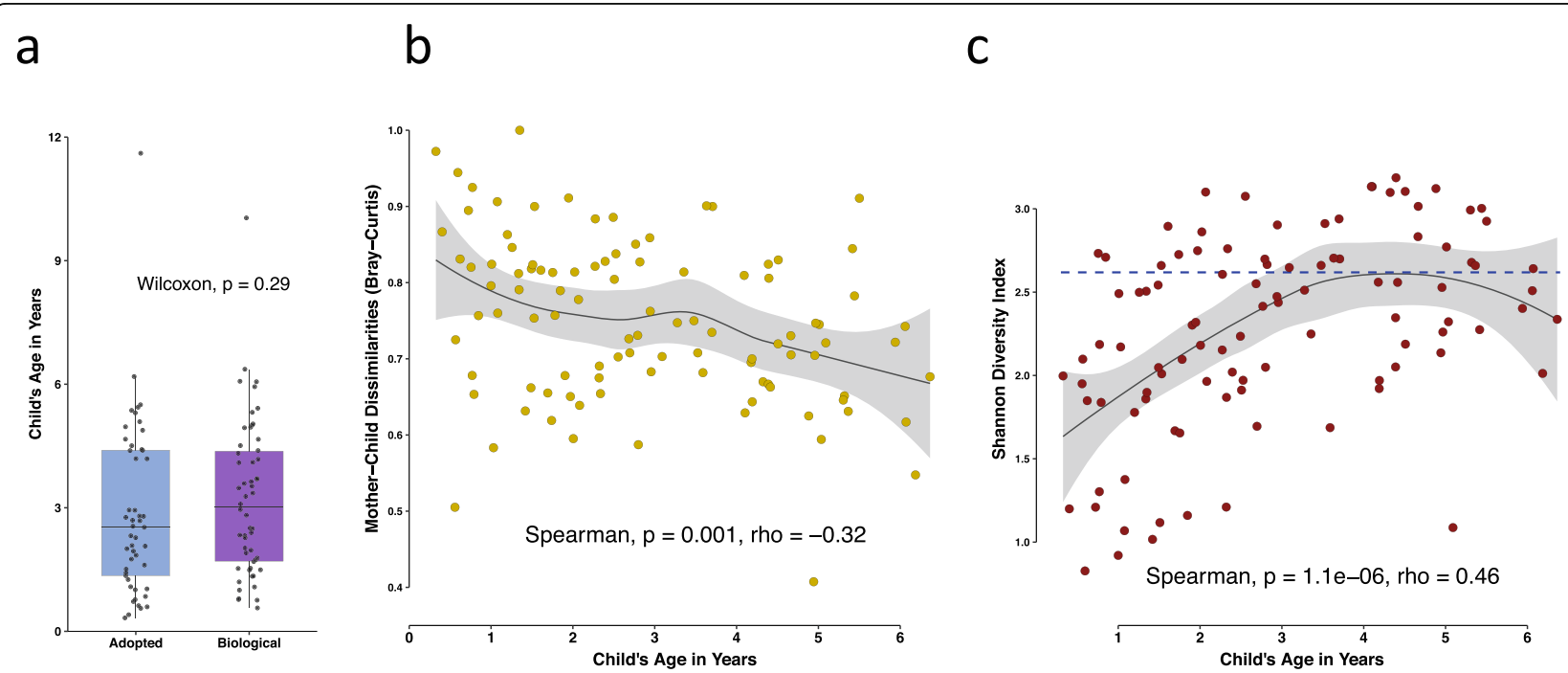

Fig. 6 Child's age was a significant determinant of mother-child dissimilarities. a Box and whisker plot showing distribution of ages among the biologic and adoptive group children. $\mathbf{b}$ Scatterplot exhibiting the relationship of mother-child distances with age of the child. A strong negative correlation between mother-child dissimilarities and child's age was observed. Both adoptive and biologic children were included in this analysis, and two older children ( $>=10$ years) were excluded $(n=101)$. c For the same 101 children, the alpha diversity measure Shannon diversity index was plotted against age. The blue dotted line represents mean Shannon diversity for mothers of those children. Alpha diversity also showed strong positive correlation with child's age, and most older children's diversities were similar to the adults. Strength and direction of associations were measured using Spearman's rank-order test. Scatter plots were smoothed using the regression method LOESS fit. Analysis was based on strain level communities 
communities were less similar to that of their mothers compared to older children (Fig. 6b). The strong negative association between child's age and mother-child dissimilarities (Spearman correlation coefficient rho $=-$ $0.33, p=0.002$ ) lead us to further explore this relationship. We hypothesized that increasing overall diversity of the oral microbial communities with age of the child may be responsible for increasing similarity of children's microbiota to their mothers with age. Indeed, our results show that the Shannon diversity index, a measure of alpha diversity, increased sharply during the early years, reaching levels comparable to those of the mothers for most children by age 5 years (Fig. 6c). Children from both the biological and adoptive groups showed the same pattern, as can be seen in Supplementary Figure S7.

\section{No influence of genetics seen for individual species}

While considering the entire bacterial community as a whole did not show any effect of genetic kinship, we wanted to explore whether individual species of bacteria showed differences in degree of strain matching between biologic and adoptive mother-child pairs. Our extensive database of oral ISR sequences allowed us to assign species level taxonomy to ISR-type strains, and a list of the most abundant species, along with the number of strains identified for each, is shown in Supplementary Table ST4. To determine if fidelity of transmission varied for individual oral bacteria species, we compared the adoptive and biologic mother-child dissimilarities from the saliva/soft tissue swab dataset for the 10 most abundant species in this dataset (Fig. 7). No species showed any significant difference between the adoptive and biologic groups, suggesting no differential heritability.

\section{Discussion}

Most investigations of the relative contribution of genetics and environment to the human microbiome have used a monozygotic vs dizygotic twin model. To our knowledge, this is the first study to directly investigate parent-to-child microbial transmission by using an adoptive versus biologic mother-child study design. Only children adopted by a genetically unrelated family were included. The genetic distances between African and northern European populations are among the greatest found in modern humans [18], and many of our

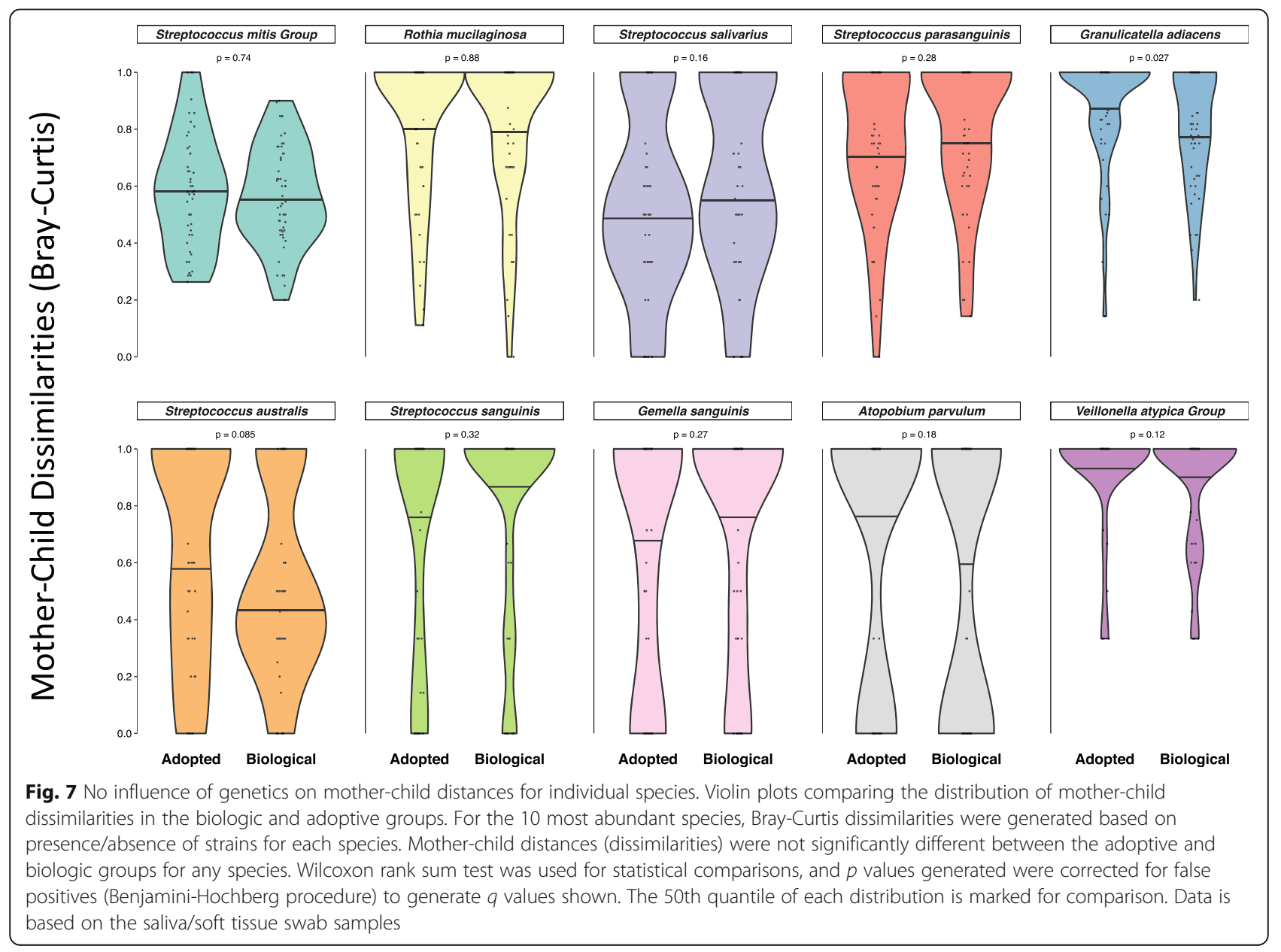


adoptive dyads were composed of white mothers and African-American children, providing maximum genetic distance between unrelated mother-child pairs. Only children adopted at birth were included to minimize contact with the birth mother's microbiota. Thus, our adoptive versus biologic study design allowed us to directly explore the contribution of host genetics to acquisition of oral microbiota. We found no measurable effect of genetic relatedness on how closely children's oral microbiota resembled that of their mothers (Fig. 3). These results suggest an alternative interpretation for the findings of two twin-based studies that have shown a small but significantly greater microbiota similarity between MZ than DZ twins $[19,20]$. What MZ twins do share to a greater extent than DZ twins and adopted siblings are important environmental determinants such as greater social closeness and intimacy, more similar treatment from others, and greater tacit coordination in choice making [21]. We suggest that the greater similarity of oral microbial communities previously observed in MZ twins as compared to DZ twins may be mediated through shared environmental factors and not a direct effect of genetically determined host factors.

Although little previous evidence is available at the strain level, studies at the species level have provided evidence that shared environment is important in shaping oral microbiota composition. In one of three published oral microbiome twin studies [19, 20, 22], MZ twin pairs were found to be no more similar than DZ twin pairs, and they became less similar when they no longer cohabited [22]. Kissing couples were observed to share highly similar tongue dorsum microbial communities [23], and shared household was more important than genetic relationship in a dataset from an extended family [24]. Another study found that household members, particularly couples, shared more of their microbiota than individuals from different households [25]. While these studies did not resolve microbial communities at strain level, and thus did not provide sufficient resolution to track transmission, they support our finding that shared environment and direct contact are the drivers of microbial community structure.

Findings from pairings within our extended biological family dataset that included fathers and siblings further supported the importance of environment over genetics in determining oral microbial community composition (Fig. 5). The pairings that showed the greatest similarity were sibling pairs and married couples, and they were equally similar despite the difference in genetic relatedness. Parent-child pairings were less similar than spouses or siblings, again pointing to degree of contact and agerelated factors as environmental determinants of microbial similarity.
Common biologic features of the mother-child relationship have been previously investigated and have been found to have some effect on microbial communities at the species level $[26,27]$. We saw no effect on the fidelity of mother-child strain sharing from vaginal birth or breast feeding (Supplementary Fig S5). We also observed that mother-child similarity was greater in older children (Fig. 6), ruling out early effects of host genetic influence that are lost over time. In addition, father-child oral microbiota matched just as closely as that of mothers and children (Fig. 5). Together, these observations suggest that any impact of breast feeding or delivery mode on oral strain sharing is negligible relative to other environmental factors.

Subgingival and supragingival niches have distinct ecologies and microbial community profiles, and dysbiosis of these communities causes the two major oral diseases, dental caries, and periodontitis [28]. The saliva and soft-tissue surfaces, being easily accessible, have been most commonly sampled, but may not reflect the disease-associated communities of greatest interest. Due to the importance of biogeographic diversity within the oral cavity [29], we sampled three distinct sites-saliva/ soft tissue surfaces, supragingival plaque biofilm, and subgingival plaque biofilm-and confirmed that the lack of a measurable effect of genetics on microbial communities was consistent across all three niches. In this study, we focused on the healthy microbiota, and future studies with larger sample sizes could address the question of disease-associated species. We collected samples at a single timepoint for this study since our previous work has shown considerable temporal stability of the oral microbiota [5].

Two separate analytic approaches were chosen to provide resolution at the level of species and strain. Specieslevel microbial identification using $16 \mathrm{~S}$ rRNA gene sequencing provides limited power to track bacterial transmission, although it may provide a good indicator of functional similarity of bacterial communities. For species-level $16 \mathrm{~S}$ OTU-based analysis, the $16 \mathrm{~S}$ V1-V3 region was targeted since it provides the best resolution for many common, closely related oral species [12]. The resulting dataset of $\sim 550$ bp paired reads, while the best choice for species-level analysis, did not lend itself to ASV level analysis with DADA2. Although ASV level analysis can often be used to increase resolution of $16 \mathrm{~S}$ datasets, the DADA2 pipeline is not designed for short overlap paired reads. It processes each pair independently, leading to low successful read pair merging when overlap is low. ISR sequencing was used for strain-level analysis. We have previously shown that this approach provides greater resolution than that of 16S ASVs [5].

Our previous work showed that individuals have relatively similar microbial profiles when analyzed at the 
species level, but their microbiota are distinct and personalized at the subspecies level [5]. For this study, we used both $16 \mathrm{~S}$ species-level and ISR strain-level sequencing approaches to compare microbial profiles. Neither approach detected a difference between adoptive and biologic dyads, but our findings illustrate the greater power of the ISR-based approach to distinguish microbial communities (Fig. 3). For example, the level of strain sharing was quite low between random pairings, only $10 \%$, while at the species level it was $41 \%$ (Fig. 4). Only the strain-level analysis consistently able to detect differences between motherchild and random pairings.

Previous studies have suggested that some taxa might be more "heritable" than others, but the findings have not been consistent across studies [19,20]. These studies used relative abundance data and interclass correlation coefficient (ICC) and ACE modeling in twins. We used presence or absence of strains in our major analyses because our question was whether children are more likely to acquire strains that have successfully established in their biological parent, and ICC would not be applicable for our binary data. We compared the frequency of strain concordance between biological and adoptive parents and offspring for the most common healthassociated species (Fig. 7). Depth of sequencing limited analysis to the 10 most abundant species. None of these showed significantly different mother-child distances when comparing adoptive to biologic groups, indicating no effect of host genetics for any of these species.

Although sample size of the present study is smaller compared to some previous studies, both our direct examination of the parent-child relationship, the greater genetic difference between dyads using adoption vs a twin model, and the strain-level resolution achieved in our study allowed us to more directly address this question.

\section{Conclusions}

The host is clearly active in shaping the composition of the oral microbiome, since fewer than a thousand of the many bacterial species in the larger environment are capable of colonizing the human oral cavity. Our findings suggest that these control mechanisms are universally shared among humans, since no effect of genetic relatedness on fidelity of microbial transmission could be detected. Instead our findings point towards contact and shared environment being the driving factors of microbial transmission, with a unique combination of these factors ultimately shaping a highly personalized human oral microbiome.

\section{Supplementary Information}

The online version contains supplementary material available at https://doi. org/10.1186/s40168-020-00986-8.
Additional file 1: Supplementary Figure S1. Strain-level community characterization led to increased separation between samples. Comparison of centroid distances for strain and species level communities, both in terms of subject type (mother/child) separation and sampling site (saliva/swab, subgingival and supragingival plaque). Samples were significantly better separated at the strain level. P-values were generated using paired Wilcoxon rank sum test (significance level ${ }^{* * *}$ refers to $p<0.001$ ). Supplementary Figure $\mathbf{S 2}$. Beta-diversity comparison among samples by subject and family types, at both species and strain levels. Non-metric multidimensional scaling (NMDS) plots using Bray-Curtis dissimilarities based on community membership, at ISRstrain level (top) and 165 Species level (bottom) are shown. Figure S3. No influence of genetics on sharing of strains between mother and child using Jaccard dissimilarities. The saliva/soft tissue swab samples were also analyzed using the Jaccard dissimilarity indices computed based on presence/absence of ISR strains. The results were very similar to what was obtained using Bray-Curtis dissimilarities. No significant difference was observed in the mother-child dissimilarities between the biologic and adoptive groups, and both biologic and adoptive children's oral microbiota were significantly more similar to their own mothers than unrelated mothers. Distribution of distances are shown using violin plots, with embedded box and whisker plots. Biological vs adoptive statistical comparisons were performed using Wilcoxon rank sum test, and related/unrelated comparisons were performed using the previously described permutation test. Figure S4. No influence of genetics on sharing of strains between mother and child using relative abundance of strains. Bray-Curtis dissimilarities between the mother-child pairs for the saliva/soft tissue swab samples were also computed based on relative abundance of ISR strains. No significant difference was observed in the mother-child dissimilarities between the biologic and adoptive groups. While the biological group children's oral microbiota was significantly more similar to their own mothers than unrelated mothers, the same distinction could not be made for the adoptive group. Biological vs adoptive statistical comparisons were performed using Wilcoxon rank sum test, and related/unrelated comparisons were performed using the previously described permutation test. Figure S5. Effect of feeding and delivery modes on mother-child distances. Differences in feeding mode (right) or delivery mode (left) among the biological group children did not have any significant effect on the motherchild dissimilarities, for either the a) saliva/soft tissue swab, b) supragingival or c) subgingival plaque samples. Figure $\mathbf{S 6}$. Extended family comparisons using plaque samples show results similar to saliva samples. Comparing microbial community similarities among different family groups, based on supragingival plaque (top) and subgingival plaque (bottom) samples from the extended biological family dataset. Groupings are ordered based on increasing median distances. Shared environment/contact lead to greater oral microbiota composition similarity, and no evidence of genetic influence was detected. Statistical comparisons were performed using Wilcoxon rank sum test and a custom permutation test (when including unrelated groups). Figure S7. Relationship of child's age with mother-child dissimilarities or alpha diversity was not different for adoptive or biological group children. a. Scatterplot exhibiting the relationship of mother-child distances with age of the child. b. Plot for Shannon Diversity Index versus child's age. The blue dotted line represents mean Shannon Diversity for mothers of those children. Strength and direction of associations were measured using Spearman's rank-order test. Scatter plots were smoothed using the regression method LOESS fit. Statistics were computed separately for the adoptive and biological group children, and two older children ( $>=10$ years) were excluded. Analysis was based on strain level communities. Supplementary Table ST1. Details of number of samples for which sequencing data was available in each group for the ISR dataset. Supplementary Table ST2. Details of number of samples for which sequencing data was available in each group for the 165 dataset. Supplementary Table ST3. List of the most widely shared species among the motherchild pairs in each group, along with their relative abundance in the dataset. Data is based on saliva/soft tissue swab samples. Supplementary Table ST4. List of the 20 most abundant oral bacteria species. Data is based on saliva/soft tissue swab samples.

Additional file 2: Supplementary Table ST5. Summary statistics table. 


\section{Acknowledgements}

Funding for this research study was provided by the National Institute of Dental and Craniofacial Research of the U.S. National Institutes of Health, under the award number R01-DE024327.

We are extremely grateful to the local adoption agencies that helped us connect with adoptive families willing to participate in this research. We are also grateful to Center of Science and Industry (COSI) Columbus for assistance with recruitment of the biologically related families. We are grateful for assistance in the laboratory from Haella Holmes, Laura Mason and Laura Cook, assistance in managing clinical data from Jodee Coldren, assistance in bioinformatic processing from Thomas Ellison and Zach Thomson.

\section{Authors' contributions}

ALG and EJL conceived the project idea and ALG designed the study. COM lead the recruitment of the adoptive families and HMS lead the recruitment and sampling of the biological families, with assistance from SBH, under the guidance of ALG. SBH and CM were responsible for the sequencing library preparation, with guidance from EJL. CM performed the bioinformatic processing and statistical data analysis, with inputs from XG and CJB, and guidance from AN and ALG. CM and ALG prepared the manuscript. All authors read and approved the final manuscript.

\section{Funding}

The research reported in this publication was supported in part by the US National Institute of Dental and Craniofacial Research, under award numbers R01 DE024327 and R01 DE024463.

\section{Availability of data and materials}

The sequencing data generated during the current study were submitted to NCBI's the Sequence Read Archive (SRA) and is accessible with SRA accession number PRJNA593844. All relevant metadata, analysis scripts, along with a reproducible workflow are available at https://github.com/cm0109/ Adoption_study. The ISR reference database is available at https://github. com/cm0109/ISR_database.

\section{Ethics approval and consent to participate}

The studies described in this manuscript were approved by the institutional review boards (IRBs) of The Ohio State University (IRB study number: 2017N0029) and Nationwide Children's Hospital (IRB study number 1400183). All participants provided informed consent for this study. Parents provided written consent and children over 7 years of age provided assent.

\section{Consent for publication}

Not applicable

\section{Competing interests}

The authors declare that they have no competing interests.

\section{Author details}

${ }^{1}$ College of Dentistry, The Ohio State University, Columbus, OH, USA.

${ }^{2}$ College of Public Health, The Ohio State University, Columbus, OH, USA.

Received: 9 March 2020 Accepted: 24 December 2020

Published online: 23 February 2021

\section{References}

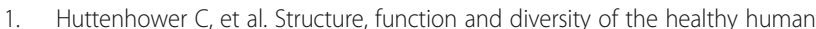
microbiome. Nature. 2012;486:207-14.

2. Dewhirst FE, et al. The human oral microbiome. J Bacteriol. 2010;192: 5002-17.

3. Sulyanto RM, Thompson ZA, Beall CJ, Leys EJ, Griffen AL. The predominant oral microbiota is acquired early in an organized pattern. Sci Rep. 2019;9: 10550.

4. Lloyd-Price J, et al. Strains, functions and dynamics in the expanded human microbiome project. Nature. 2017;550:61-6.

5. Mukherjee C, Beall CJ, Griffen AL, Leys EJ. High-resolution ISR amplicon sequencing reveals personalized oral microbiome. Microbiome. 2018;6:153.

6. Faith JJ, et al. The long-term stability of the human gut microbiota. Science (80- ). 2013;341:1237439.
7. Ramos-Gomez F, Ng M-W. Into the future: keeping healthy teeth caries free: pediatric CAMBRA protocols. J Calif Dent Assoc. 2011;39:723-33.

8. Si J, Lee S, Park JM, Sung J, Ko GP. Genetic associations and shared environmental effects on the skin microbiome of Korean twins. BMC Genomics. 2015;16:992.

9. Zaura E, Keijser BJ, Huse SM, Crielaard W. Defining the healthy 'core microbiome' of oral microbial communities. BMC Microbiol. 2009;9:259.

10. Weisburg WG, Barns SM, Pelletier DA, Lane DJ. 16S ribosomal DNA amplification for phylogenetic study. J Bacteriol. 1991;173:697-703.

11. Schloss PD, et al. Introducing mothur: open-source, platform-independent, community-supported software for describing and comparing microbial communities. Appl Environ Microbiol. 2009;75:7537-41.

12. Griffen $A L$, et al. CORE: a phylogenetically-curated 165 rDNA database of the CORE oral microbiome. PLoS One. 2011;6:1-10.

13. Callahan BJ, et al. DADA2: high-resolution sample inference from Illumina amplicon data. Nat Methods. 2016;13:581-3.

14. Oksanen J, et al. Vegan: community ecology package; 2018.

15. Kassambara A. Ggpubr: 'ggplot2' based publication ready plots; 2018.

16. Reiss PT, Stevens MHH, Shehzad Z, Petkova E, Milham MP. On distancebased permutation tests for between-group comparisons. Biometrics. 2010; 66:636-43.

17. Wickham $\mathrm{H}$, et al. ggplot2: create elegant data visualisations using the grammar of graphics; 2019.

18. Tierney BT, et al. The landscape of genetic content in the gut and oral human microbiome. Cell Host Microbe. 2019;26:283-295.e8.

19. Gomez A, et al. Host genetic control of the oral microbiome in health and disease. Cell Host Microbe. 2017;22:269-278.e3.

20. Demmitt BA, et al. Genetic influences on the human oral microbiome. BMC Genomics. 2017;18:1-15.

21. Segal NL, McGuire SA, Miller SA, Havlena J. Tacit coordination in monozygotic twins, dizygotic twins and virtual twins: effects and implications of genetic relatedness. Pers Individ Dif. 2008;45:607-12.

22. Stahringer SS, et al. Nurture trumps nature in a longitudinal survey of salivary bacterial communities in twins from early adolescence to early adulthood. Genome Res. 2012;22:2146-52.

23. Kort $R$, et al. Shaping the oral microbiota through intimate kissing. Microbiome. 2014:2:41.

24. Shaw $L$, et al. The human salivary microbiome is shaped by shared environment rather than genetics: evidence from a large family of closely related individuals. MBio. 2017;8:e01237-17.

25. Song SJ, et al. Cohabiting family members share microbiota with one another and with their dogs. Elife. 2013;2013:1-22.

26. Holgerson PL, et al. Oral microbial profile discriminates breast-fed from formula-fed infants. J Pediatr Gastroenterol Nutr. 2013:56:127-36.

27. Lif Holgerson P, Harnevik L, Hernell O, Tanner ACR, Johansson I. Mode of birth delivery affects oral microbiota in infants. J Dent Res. 2011;90:1183-8.

28. Wade WG. The oral microbiome in health and disease. Pharmacol Res. 2015; 69:97-114.

29. Mark Welch JL, Dewhirst FE, Borisy GG. Biogeography of the oral microbiome: the site-specialist hypothesis. Annu Rev Microbiol. 2019;73: 335-58.

\section{Publisher's Note}

Springer Nature remains neutral with regard to jurisdictional claims in published maps and institutional affiliations.

\section{Ready to submit your research? Choose BMC and benefit from:}

- fast, convenient online submission

- thorough peer review by experienced researchers in your field

- rapid publication on acceptance

- support for research data, including large and complex data types

- gold Open Access which fosters wider collaboration and increased citations

- maximum visibility for your research: over $100 \mathrm{M}$ website views per year

At $\mathrm{BMC}$, research is always in progress.

Learn more biomedcentral.com/submissions 